\title{
Effects of high intensity deadlift on standing long jump ability
}

\author{
Teag Whiting ${ }^{1}$, Alan Bishop ${ }^{2}$, Chad Harris ${ }^{3}$, Joseph Berning ${ }^{4}$, Kent J. Adams 5 , \\ Mark DeBeliso ${ }^{1}$
}

\author{
${ }^{1}$ Department of Kinesiology and Outdoor Recreation, Southern Utah University, Cedar City, UT, USA. \\ ${ }^{2}$ Athletics Department, Utah State University, Logan, UT, USA. \\ ${ }^{3}$ College of Professional Studies, Metropolitan State University of Denver, Denver, CO, USA. \\ ${ }^{4}$ Department of Kinesiology and Dance, New Mexico State University, Las Cruces, NM, USA. \\ ${ }^{5}$ Kinesiology Department, California State University Monterey Bay, Seaside, CA, USA.
}

\begin{abstract}
Post activation potentiation (PAP) is an acute enhancement of muscular function subsequent to an intense muscle conditioning activity. Practical application of PAP as a physical preparation for enhanced performance is a topic of considerable interest in the sport performance community. The purpose of this study was to determine if a dynamic warm-up (WU) plus a progression of deadlift (DL) sets culminating in a one-repetition maximum DL (1-RM DL) (i.e., a PAP conditioning activity) is more effective in improving the standing long jump (SLJ) as opposed to using a dynamic WU alone. Secondarily, it was of interest to determine if there was a meaningful relationship between the 1-RM DL and SLJ ability. Twenty NCAA Division I male North American football players participated in the study (age $=20.3 \pm 2.3$ years, height $=183.5 \pm 6.3 \mathrm{~cm}$, mass $=96.3 \pm 17.1 \mathrm{~kg}$ ). Participants met three times separated by one week. The initial gathering was an orientation meeting where participants were briefed on the study protocol and height, age and body mass were recorded. During the first data collection the participants completed a dynamic WU immediately followed by a progression of DLs culminating in a 1-RM DL; this served as a PAP conditioning activity. Following the PAP WU (5-6 minutes) the participants completed two trials of SLJs separated by 2-3 minutes. During the second data collection the participants completed the dynamic WU only followed (5-6 minutes) by the participants completing two trials of SLJs separated by 2-3 minutes. A paired t-test was used to compare the maximum SLJ between WU conditions and a Pearson correlation coefficient ( $r$ ) was calculated to compare 1-RM DLs with SLJ scores. The SLJ was significantly greater $(4.3 \pm 7.5 \mathrm{~cm})$ following the 1-RM DL PAP warm-up $(p<0.01)$. The
\end{abstract}

relationship between the 1-RM DL and SLJ scores was $r=0.53$ $(p<0.01)$ with a coefficient of determination of $C D=0.28$. Within the parameters of this study, a dynamic WU that included a 1-RM DL as a PAP conditioning activity significantly improved SLJ performance over a dynamic WU alone. Further, there is a moderate positive relationship between maximum DL performance and SLJ ability.

Keywords. NCAA football, post activation potentiation, standing broad jump.

\section{Introduction}

$\mathrm{P}$ ost activation potentiation (PAP) is defined as an acute enhancement of muscle function following an intense muscle activity (Hodgson, Docherty, \& Robbins, 2005). Described another way, PAP refers to the increased neuromuscular state that occurs immediately following a high intensity exercise known as the PAP conditioning activity (Anthi et al., 2014; Robbins, 2005). The theoretical mechanisms considered responsible for the PAP phenomena have been elucidated elsewhere (NSCA Hot Topic, 2016). However the practical applications of PAP remain a topic of contemporary research (NSCA Hot Topic, 2016). Bishop's (2008) applied research model for the sport sciences strongly encourages the pursuit of meaningful applied research for sport performance enhancement. 
As the athletic community is becoming increasingly aware of the potential performance benefits of PAP the resounding question could be described as: are there practical ways to use PAP to enhance athletic performance? In this regard, the barbell deadlift (DL) is a closed chained full body movement that is known for its' potential for developing full body strength (O'Shea, 2000) and is relatively simple to learn and execute. The DL is a whole body structural exercise that lays a foundation for success (Fleck \& Kraemer, 2004). A detailed description of the execution and benefits of the DL has been described previously (O'Shea, 2000) and as such would seem a potentially good choice as a PAP conditioning activity. However, a recent study by Arias, Coburn, Brown, \& Galpin (2016) was unsuccessful utilizing the DL as a conditioning activity for the enhancement of the vertical jump. Respectfully, we found the results of the aforementioned study to be counter intuitive. Hence, in the spirit of the article by Open Science Collaboration (2015) regarding reproducibility, a "defining feature of science", we choose to reinvestigate the DL as a potential PAP modality for enhancing physical performance.

The standing long jump (SLJ) is a full-body coordinated movement driven by lower-body muscle power (Ah Sue et al., 2016). Additionally, the SLJ is a relatively easily administered test, feasible in a variety of settings, with basically no cost (i.e., even a reliable vertical jump test requires equipment costing a few hundred dollars) (Ah Sue et al., 2016). The aforementioned study by $\mathrm{Ah}$ Sue et al. (2016) demonstrated that a barbell back squat was effective as a PAP conditioning activity for the enhancement of the SLJ. While the back squat is not identical to the deadlift, it is somewhat biomechanically similar as both the DL and back squat stress the gluteal maximus, semimembranosus, semitendinosus, biceps femoris, vastus lateralis, vastus intermedius vastus, vastus medialis, and rectus femoris (Baechle \& Earle, 2008). Further, both the back squat and the DL challenge the muscle groups that comprise what is referred to as the "core" which includes the diaphragm, latissimus dorsi, the abdominal wall, the pelvic floor, the paraspinals , the hip girdle, and the quadratus lumborum (HuxelBliven \& Anderson, 2013; Kibler et al., 2006).

Given the biomechanical similarity between the back squat and the DL, the failure of previous research at establishing the success of utilizing the DL as a PAP conditioning activity, the simplicity of learning and executing the DL, and the low cost and ease of administration of the SLJ, we choose to formulate a study examining the effects of a PAP DL conditioning activity on SLJ performance.

Hence, the purpose of this study was to determine if a dynamic warmup (WU) plus a progression of DL sets culminating in a maximum 1-RM DL (PAP conditioning activity) is more effective in improving the SLJ as opposed to using a dynamic warm up (WU) alone. This study also aimed to determine if there is a meaningful correlation between (1-RM) DL and SLJ scores in collegiate North American football players.

It is hypothesized that warming up with a progression of DLs leading to a 1-RM DL will be effective as a PAP stimulus resulting in an increase of SLJ performance. Further, we anticipate a moderate to high correlation between 1-RM DL and SLJ scores.

\section{Methods}

\section{Participants}

The participants for this study were a convenience sample of male athletes from Southern Utah University's North American Football team. Permission from the head coach was sought and granted prior to the study. The athletes were then invited to volunteer to participate in the study. Permission from the University Institutional Review Board (IRB) for the use of human subjects in research was obtained prior to conducting any assessments of the participants. Further, the participants were provided a written consent form (IRB approved) to read and sign before the study was engaged. Participating athletes were informed of the risks involved and that participation was completely voluntary. They were also informed that at any time, if they wished to discontinue participation in the study, they could do so without penalty. All of the participants were at least 18 years old.

All athletes were experienced and proficient in both the SLJ and DL exercise. By using trained and experienced athletes we hoped to maximize the potential benefits of the PAP WU's, while minimizing the effects of learning between testing procedures. All participants had been trained by the Strength and Conditioning Staff, therefore all athletes had prior 
training for at least one preseason and up until the point of the study. The study took place during the football competitive season acknowledging that weekly fluctuations in fatigue could impact the study outcome. This team was selected because strength and power are attributes that are very beneficial to football players, and this study outcome may benefit both attributes. Only athletes who were free of injury were allowed to participate. Participants were asked to not alter their normal nutrition and hydration routines during the test period.

\section{Instruments and Apparatus}

The study sessions were conducted in the Weight Room at the Athletic Complex at Southern Utah University (Figure 1). Equipment necessary to collect the 1-RM DLs in this study included a $20.45 \mathrm{~kg}$ barbell and weighted plates (ranging from 1.14-20.45 kgs) housed in the Athletics' weight room. The SLJ measures required the use of the measuring tape, blue painter masking tape to set up jump markers, and a measuring stick.

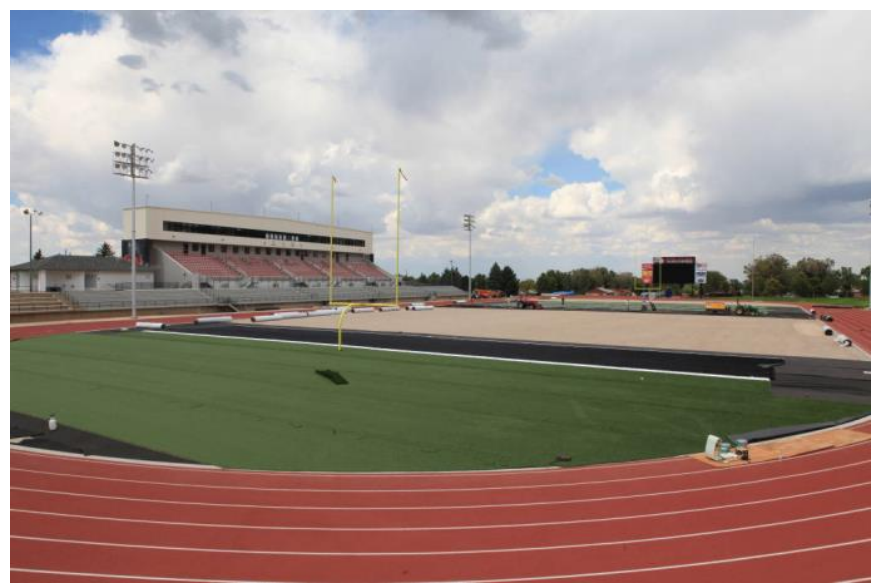

Figure 1. Southern Utah University Athletics Facility.

\section{Procedures}

Participants met for an initial familiarization period and two data collection sessions (separated by one week). During the familiarization period the participant's height, mass, and age were collected. Subsequently they were instructed on the WU procedures for the following two sessions and subsequent DL and SLJ testing procedures.
During the first data collection day a warmup (WU) using a using a progression of conventional style DL sets culminating in a maximum 1-RM DL (PAP conditioning activity) was conducted. Prior to the progression of DL sets, a dynamic WU was conducted (described below). The conventional DL was conducted using standards outlined by the NSCA (Baechle \& Earle, 2008) in which weight is lifted from the floor to an erect position, and lowered back to the floor. An alternated (over/under grip) was used for all the DL WU progressions and 1-RM attempts. The DL WU progressions to achieve the 1-RM DL were as follows (and as described by Bishop et al., 2014). "A warm-up set of 5-10 repetitions was performed using $40-60 \%$ of the estimated 1-RM. After a 2-5 minute rest period, a set of three repetitions was performed at 60 $90 \%$ of the estimated 1-RM. Then, after a 5 minute rest, 3-4 maximal attempts were performed to determine the 1-RM. Rest periods between trials lasted 2-5 minutes. A complete range of motion and proper technique was required for a lift to be considered successful (i.e., each subject had to complete the repetition to full trunk extension; and they were not allowed to continue testing once a weight was reached that caused technical breakdown, which was considered head and shoulders tilting over the feet or little to no knee bend during extension)". Following the completion of the 1-RM DL (PAP conditioning activity) (5-6 minutes) the participants proceeded to have the SLJ measures collected as described below.

Each participant was provided 2-3 practice SLJ trials. Following the practice trials the participants performed two trials with the best score being recorded for statistical analysis. Test trials were separated by 2-3 minutes. The participants assumed a standstill position with tip of the shoe just touching the starting line (Baechle \& Earle, 2008). The participant then performed the SLJ, specifically, a counter-movement jump, bounding as far forward as possible (Baechle \& Earle, 2008). A mark was placed at the back edge of the participant's rear most shoe heel, and the tape measure determined the distance between the starting line and the mark (Baechle \& Earle, 2008). For the jump to be scored the participant was required to land on both feet (Baechle \& Earle, 2008). In the event a participant faulted on the attempt they were allowed only one immediate re-trial (hence avoiding fatigue or interrupting another participant's attempts. 


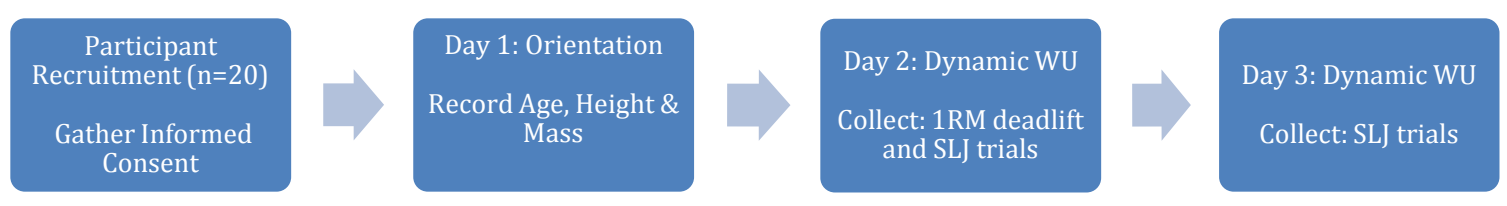

Figure 2. Study time line overview of events (WU-warm-up; 1RM-one repetition maximum; SLJ-standing long jump).

During the second data collection day a dynamic WU was conducted comprised of low intensity drills that the participants were acquainted with (10 jumping jacks, 10 cross jacks, 10 squat hops, 10 quick hops, 10 quick jumps, 10 lunges (5 each leg), 10 push-ups, 10 sit ups, 10 Chris Farleys, 10 arm circles forward, 10 arm circles backward, and 10 jump tucks). This was the same dynamic WU conducted prior to the DL PAP conditioning activity (described above). Following the dynamic WU (5-6 minutes) two SLJ trials were collected in the identical manner as described above. The goal of the dynamic WU was to increase blood flow and body temperature as well as provide sport specific drills to prepare the athletes for the upcoming SLJ trials (Baechle \& Earle, 2008).

The test administrator was a trained strength professional with knowledge and experience in performing and teaching the conventional DL. The test administrator monitored the dynamic WUs, each DL progression, as well as the SLJ trials. It should also be noted that testing took place the same time of day and the participants were requested not to alter their typical nutrition intake and hydration patterns during the study period.

\section{Reliability}

The reliability of the SLJ has been reported to range from ICC=0.89-0.97 (Ah Sue et al., 2017) in female collegiate volleyball players and an ICC $=0.93$ in physically active college age males (Markovic et al.,
2004). The National Strength and Conditioning Association recognize $1 \mathrm{RM}$ and $3 \mathrm{RM}$ measures as reliable measures of muscular strength (Baechle \& Earle, 2008). Further, reported reliability coefficients $(r \geq 0.90$ and ICC $\geq 0.90)$ confirm that $1 R M$ s and $3 R M s$ are very reliable measures (McCurdy et al., 2004; Tagesson \& Kvist, 2007).

\section{Design and Analysis}

The dependent variable analyzed in this study was the SLJ following two WU conditions (dynamic WU and a dynamic WU plus a 1-RM DL PAP conditioning event). There were two trials of SLJ scores collected following each WU condition. A dependent t-test was used to compare the SLJ scores following the two WU conditions. Specifically, the maximal trial SLJ score following the dynamic WU was compared to the maximal trial 2 SLJ score following the 1-RM DL PAP conditioning event. The statistical significance for this study was $\alpha \leq 0.05$, however $p$-values near the 0.05 were considered potentially meaningful with respect to practical applications.

\section{Results}

A total of 20 athletes participated in the study and their demographics are reported in Table 1. All participants completed the study without incidence. Also reported in Table 1 are the participant 1-RM deadlift scores as well as the deadlift scores normalized to the participant's body mass.

Table 1

Participant descriptive information $(n=20)$.

\begin{tabular}{lccccc}
\hline $\mathrm{N}$ & Age (years) & Height $(\mathrm{cms})$ & Mass $(\mathrm{kgs})$ & 1-RM DL $(\mathrm{kgs})$ & 1-RM DL/BM \\
\hline 20 & $20.3 \pm 2.3$ & $183.5 \pm 6.3$ & $96.3 \pm 17.1$ & $188.0 \pm 21.9$ & $2.0 \pm 0.3$ \\
\hline $\begin{array}{l}\text { Note }{ }^{1}: \\
\text { maximum deadlicipant means }\end{array}$ & and standard deviations for descriptive information. 1-RM DL-one repetition & DL/BM- one repetition maximum deadlift/body mass.
\end{tabular}


Table 2

Standing long jump scores.

\begin{tabular}{lccc}
\hline & Dynamic WU $(\mathrm{cms})$ & PAP WU DL $(\mathrm{cms})$ & SLJ $(\mathrm{cms}) \Delta$ \\
\hline Best Trial Score & $257.3 \pm 23.7$ & $261.6 \pm 24.4^{*}$ & $4.3 \pm 7.5$ \\
\hline $\begin{array}{l}\text { Note }^{1}: \text { Mean } \pm S D, \text { WU-warmup, } P A P \\
\text { between } W U \text { Conditions. Note }{ }^{2}:{ }^{*} \text { significant } p<0.001\end{array}$ & &
\end{tabular}

Table 3

Individual 1-RM deadlift (kgs) and standing long jump scores (cms).

\begin{tabular}{|c|c|c|c|c|c|}
\hline Participant & DL 1-RM & $\begin{array}{c}\text { DL 1-RM/Body } \\
\text { Mass }\end{array}$ & $\begin{array}{c}\text { PAP DL WU } \\
\text { SLJ }\end{array}$ & $\begin{array}{c}\text { Dynamic WU } \\
\text { SLJ }\end{array}$ & $\% \Delta$ \\
\hline 1 & 165.9 & 1.9 & 266.7 & 264.2 & 1.0 \\
\hline 2 & 197.7 & 2.5 & 281.9 & 279.4 & 0.9 \\
\hline 3 & 165.9 & 2.0 & 276.9 & 269.2 & 2.8 \\
\hline 4 & 184.1 & 2.0 & 309.9 & 299.7 & 3.4 \\
\hline 5 & 193.2 & 1.9 & 261.6 & 256.5 & 2.0 \\
\hline 6 & 197.7 & 1.6 & 264.2 & 256.5 & 3.0 \\
\hline 7 & 211.4 & 2.4 & 274.3 & 281.9 & -2.7 \\
\hline 8 & 184.1 & 2.1 & 266.7 & 261.6 & 1.9 \\
\hline 9 & 215.9 & 2.8 & 264.2 & 256.5 & 3.0 \\
\hline 10 & 197.7 & 1.5 & 205.7 & 203.2 & 1.3 \\
\hline 11 & 147.7 & 1.8 & 274.3 & 261.6 & 4.9 \\
\hline 12 & 136.4 & 1.6 & 243.8 & 256.5 & -5.0 \\
\hline 13 & 229.5 & 1.9 & 254.0 & 261.6 & -2.9 \\
\hline 14 & 229.5 & 1.8 & 231.1 & 215.9 & 7.1 \\
\hline 15 & 165.9 & 2.0 & 287.0 & 276.9 & 3.7 \\
\hline 16 & 170.5 & 2.0 & 289.6 & 276.9 & 4.6 \\
\hline 17 & 197.7 & 2.0 & 243.8 & 233.7 & 4.3 \\
\hline 18 & 156.8 & 1.4 & 221.0 & 221.0 & 0.0 \\
\hline 19 & 147.7 & 1.6 & 262.9 & 266.7 & -1.4 \\
\hline 20 & 206.8 & 2.3 & 251.5 & 246.4 & 2.1 \\
\hline
\end{tabular}

Note $^{1}$ : WU-warmup, PAP WU DL-PAP Warm Up with the deadlift, \% change-percent increase of SLJ following PAP DL WU.

\section{Discussion}

The purpose of this study was to determine if a dynamic WU that included a 1-RM DL as a PAP conditioning activity was more effective at improving SLJ scores as opposed to a dynamic WU alone. Further, it was of interest to determine if there was a meaningful relationship between SLJ scores and the 1-RM DL normalized to body mass. It was hypothesized that the dynamic WU consisting of a DL as a PAP conditioning activity would be superior to the dynamic WU and that there would be a meaningful relationship between the SLJ scores and the 1-RM DL/body mass. In support of our research hypotheses, the dynamic WU that included a 1-RM DL as a PAP conditioning activity led to significantly greater SLJ scores than the SLJ scores following the dynamic WU alone $(p<0.01)$. Additionally, there was a moderate positive 
relationship between the SLJ scores and the 1-RM DL/body mass $(\mathrm{p}<0.01)$.

The average SLJ scores following the PAP WU were $261.6 \pm 24.4 \mathrm{~cm}$ which were approximately $65 \%$ ile for NCAA Division I North American football offensive line players competing at the NFL combines (Hoffman, 2006). The average 1-RM DL recorded in this study $(188.0 \pm 21.9 \mathrm{~kg})$ was considered as intermediateadvanced for 18-23 year old males with $95 \mathrm{~kg}$ body mass (StrengthLevel.Com, 2017).

The improvement in SLJ performance as a result of implementing a PAP WU is consistent with previous studies which have demonstrated that a PAP warm-up can significantly increase both upper and lower body power output (Harris et al., 2004; Harris et al., 2006; Mallander et al., 2006; Berning et al., 2010; Harris et al., 2011; Dove et al., 2013; Hamilton et al., 2016; Ah Sue et al., 2016, Tano et al., 2016).

Fifteen (or $75 \%$ ) of the 20 participants experienced an improvement in SLJ performance while the others experienced no change $(5 \%)$ or a slower time $(20 \%)$ as the result of the PAP warm-up. The improvement in SLJ scores of the participants who experienced an increase ranged from $2.5-15.2 \mathrm{~cm}$ (or 1.0-7.1\%) and averaged $7.8 \pm 4.0 \mathrm{~cm}$ (or $3.1 \pm 1.7 \%$ ). Research regarding the effects of a PAP WU strategy indicates that the training status of an athlete is a primary factor necessary for a PAP WU to be successful (Gullich \& Schmidtbleicher, 1996; Hrysomallis \& Kidgell, 2001; Gilbert \& Lees, 2005; Kilduff, et al., 2007). The National Strength and Conditioning Association (NSCA) states that PAP WU protocols should be "reserved for resistance-trained power athletes with high relative strength" (NSCA Hot Topics, 2016). The participants in this study were NCAA North American football players that had relatively high DL strength to body mass ratios $(2.0 \pm 0.3)$ and had a meaningful background performing the conventional DL. Hence, the participants in this study did meet the NSCA's PAP recommendations. As such, prior training status and relative strength may have been partly responsible for the majority of the participants experiencing a measureable potentiation as a result of the PAP DL protocol used in this study. Conversely, it appears that the training status and relative strength did not facilitate five of the participants (or 25\%) from experiencing a potentiating effect. It is possible that for these 5 individuals who did not experience a potentiating effect that the DL potentiating activity induced to great of a fatigue state.

Whilst the results of our study confirmed our research hypothesis, the results were in conflict with the results of recent study by Arias et al. (2016) that was not successful at inducing a measureable PAP effect on vertical jump performance as the result of a PAP WU strategy that also employed the DL as the conditioning activity. We suspect that the primary reasons for the dichotomy in results between the Arias et al. (2016) results and the current study may be due to two factors: intensity of the conditioning activity and recovery time. The intensity of the PAP DL conditioning activity in the Arias et al. protocol was 5repetitions of the DL at $85 \%$ of $1-\mathrm{RM}$ while the stimulus in the current study was a 1-RM DL preceded by the progression of WU sets to establish the 1-RM. The recovery time between the PAP conditioning activity and the vertical jumps in the Arias et al. study ranged from 15 seconds to 16 minutes post, in 2 minute intervals. . The current study used a recovery time of 59 minutes to conduct two SLJ trials, which may have attenuated potential fatigue. In fact, Arias et al. suggest a short recovery time may potentially explain their results. Additionally, one must consider that despite biomechanical similarities, the SLJ and vertical jump are two different movements that may respond differently to the same PAP conditioning movement (in this case the DL).

Another possible explanation between the findings of the current study and the Arias et al., 2016 study has to do with muscle fiber composition. Individuals with a higher fast twitch muscle fiber composition are more likely to experience a positive outcome as the result of a PAP conditioning activity (Hamada et al., 2000). The participants in the Arias et al. study were noted as being recreationally trained men, which provided little insight into their muscle fiber composition. However, the participants in the current study were NCAA Division I North American football players; a sport that almost requires a muscle fiber composition that is predominately fast twitch. Fast twitch muscle fibers are hypothesized to undergo a higher degree of phosphorylation in response to a PAP excitation event leading to a greater potentiation than slow twitch fibers (Metzger et al., 1989; Moore \& Stull, 1984).

The realization of a positive PAP protocol is anchored in finding the optimal stimulus that allows 
for the coexistence (or minimizing) of fatigue while the muscles are in a potentiated state (Rassier \& MacIntosh, 2000). Frankly, we were encouraged at the positive findings that a maximal effort (1-RM) did not lead to a fatigue state that would be too great to experience a PAP effect (i.e. lack of coexistence). It could be that the low volume in the presence of high intensity was responsible for the positive results of the current study and represent a new paradigm of study in terms of finding the ideal stimulus to induce a positive PAP effect.

In previous research regarding PAP it has been suggested that a potentiating period of 2-20 minutes exists following the PAP conditioning activity (NSCA Hot Topics, 2016) with the optimal muscle potentiation occurring at 10 minutes (Ah Sue et al., 2016). The SLJ trials collected in the current study were at 5-6 and again at 7-9 minutes following the PAP conditioning activity. As such, we felt that the timing of collecting the SLJ trials was inside of the PAP potentiation window and close to the optimal potentiation suggested by Ah Sue et al., 2016. While the current study was successful at inducing a PAP effect with aforementioned rest period leading to the collection of the SLJ trials, we agree with Hamilton et al., 2015 in "that the rest period to be employed in a PAP protocol should be developed on an individual basis considering the training status of the athlete as well as how the individual feels on a certain day (sleep, nutritional status, life stresses, etc.)."

This study also examined the relationship between the 1-RM DL/body mass and SLJ. The Pearson correlation coefficient $(\mathrm{r})$ between the 1-RM DL/body mass and the maximum SLJ scores was $r=0.53(p<0.01)$. The coefficient of determination $\left(C D=r^{2}\right)$ was $C D=0.28$. The positive PCC between the 1-RM DL/body mass and the maximum SLJ scores is considered moderate (Safrit \& Wood, 1995) and the $C D=0.26$ indicates that $26 \%$ 1-RM DL and the maximum SLJ come from common factors (Safrit \& Wood, 1995). The PCC between the 1-RM DL/body mass and SLJ was a positive and moderate $\mathrm{r}=0.56 \mathrm{p}<0.05$ ). In other words, a low percentage of physical attributes that contribute to the performance in the 1-RM DL/body mass also contribute to the performance in the SLJ. Given that the DL is a strength movement and the SLJ is a power movement it seems reasonable that such a low CD would exist. Given that the $\mathrm{CD}$ is so low, it is impressive that using the 1-RM DL as a PAP conditioning activity could lead to such a meaningful potentiating effect on SLJ scores.

We felt a strength of this study was that we were able to work with a collegiate athletes with a reasonable sample size $(n=20)$. However, a larger sample size would have provided an additional level of assurance to the robustness of the results (assuming the statistical analysis was unchanged). A weakness of the current study was that we were unable to employ a cross-over design. Logistics of working with the football team (limited access to the team and flexibility of facility space) prevented us from a superior study (i.e. cross-over design). However, the SLJ trials following the dynamic WU only were conducted during the second data collection. If there was a learning effect, it would have favored these non-PAP WU SLJ scores, which lends credence to the results of the current findings. A second weakness of the study is the number of SLJ trials collected following the two different WU strategies. In reflection, we could have attempted to collect at least four more trials following each WU strategy to get a feel for the duration of time the potentiating effect lasts given the parameters employed in this study.

Whilst "SLJ is a full-body coordinated movement driven by lower-body muscle power" (Ah Sue et al., 2016), it is not possible to know if improvements in SLJ ability as a result of a PAP WU would be specific and transferable to the unpredictable factors associated with a North American football game. Nevertheless, it may be reasonable to think that an elevated neuromuscular state induced by a PAP WU might well lead to a slight edge over the competition for the early minutes of football game. Whilst plays occurring at the end of the game gather the most attention, we agree with Tano et al., 2016 in that "all plays count equally". Implementing a pregame PAP WU strategy that could win the early plays of the game could establish the momentum for the entire game. Further, we would suggest that it may be possible to use a PAP warm-up just prior to the beginning of the second half of the game in hopes of gaining an edge for the start of the third quarter. Due to the practical nature of the DL (i.e., one could easily have a barbell and plywood platform on the sidelines), we suggest testing the real-world potential of a PAP WU strategy during spring scrimmage of a football game. 


\section{Conclusion}

Within the parameters of this study it is concluded that;

- using a dynamic WU that includes a PAP DL is a viable way to improve SLJ performance over a dynamic WU alone; further,

- there is a moderate positive relationship between 1-RM DL ability and SLJ performance, and,

- bridging the gap between controlled PAP research and sport competition application should be a priority for researchers interested in sport performance.

\section{Conflict of Interest Declaration}

No funding was received for this research. The authors have no conflict of interest related to this research.

\section{Acknowledgements}

The authors thank Southern Utah University Athletics for their role in making this successful study possible.

\section{References}

Ah Sue R, Adams KJ, DeBeliso M. Optimal timing for post-activation potentiation in women collegiate volleyball players. Sports, 2016; 4(2): 27.

Ah Sue R, Harris C, Berning J, Sevene T, Adams KJ, DeBeliso M. Determination of trials needed for measurement consistency of standing long jump in female collegiate volleyball athletes: A brief report. International Journal of Sports Science, 2017; 7(1): 1-5.

Anthi X, Dimitrios P, Christos K. On the mechanisms of postactivation potentiation: the contribution of neural factors. Journal of Physical Education \& Sport, 2014; 14(2): 134-137.

Arias JC, Coburn JW, Brown LE, Galpin AJ. The acute effects of heavy deadlifts on vertical jump performance in men. Sports, 2016; 4: 22.

Baechle TR, Earle RW. Essentials of strength training and conditioning (3rd ed.). Champaign, IL: Human Kinetics, 2008.

Bishop A, DeBeliso M, Sevene TG, Adams KJ. Comparing 1RM and 3RM between conventional and eccentrically loaded deadlifts. J Strength Cond Res, 2014; 28(7): 1820-1825.

Bishop D. An applied research model for the sport sciences. Sports Med, 2008; 38(3): 253-263.
Berning JM, Adams KJ, DeBeliso M, Sevene-Adams PG, Harris C, Stamford BA. Effect of functional isometric squats on vertical jump in trained and untrained men. J Strength Cond Res, 2010; 24(9): 2285-2289.

Dove BC, Sevene TG, Harris C, DeBeliso M, Adams KJ, Carson CA, Berning JM. 5RM Bench press increases medicine ball put distance in untrained males but not untrained females. Med Sci Sport Exer, 2013; 45(5): S597.

Fleck SJ, Kraemer WJ. Designing resistance training programs. Champaign, IL: Human Kinetics, 2005.

Gilbert G, Lees A. Changes in the force development characteristics of muscle following repeated maximum force and power exercise. Ergonomics, 2005; 48: 1576-1584.

Guellich A, Schmidtbleicher D. MVC-induced short-term potentiation of explosive force. New Studies in Athletics, 1996; 11(4): 67-81.

Hamada T, Sale DG, MacDougall JD, Tarnopolsky MA. Postactivation potentiation, fiber type, and twitch contraction time in human knee extensor muscles. J Appl Physiol, 2000; 88(6): 2131-2137.

Hamilton C, Berning JM, Sevene TG, Adams KJ, DeBeliso M. The effects of post activation potentiation on the hang power clean. Journal of Physical Education Research, 2016; 3(1): $1-9$.

Harris C, Dolny D, Browder K, Adams KJ, DeBeliso M. The effect of prior contractile activity on power output. Med Sci Sport Exer, 2004; 36(5): S2379.

Harris C, Kipp K, Adams KJ, DeBeliso M, Berning JM. The effects of high intensity warm-up on shot put performance. Med Sci Sport Exer, 2011; 43(5): S600.

Harris C, Moore J, DeBeliso M, Adams KJ, Berning J, Hansen C. The effects of heavy squats on potentiating 30 second Wingate test performance. Med Sci Sport Exer, 2006; 38(5): S292.

Hoffman J. Norms for fitness, performance, and health. Human Kinetics, Champaign, IL, USA, 2006.

Hodgson M, Docherty D, Robbins D. Post-activation potentiation. Sports Medicine, 2005; 35(7): 585-595.

Hrysomallis C, Kidgell D. Effect of heavy dynamic resistive exercise on acute upper-body power. J Strength Cond Res, 2001; 15: $426-430$.

Huxel-Bliven KC, Anderson BE. Core stability training for injury prevention. Sports Health: A Multidisciplinary Approach, 2013; 5(6): 514-522.

Kibler WB, Press J, Sciascia A. The role of core stability in athletic function. Sports Med, 2006; 36(3): 189-198.

Kilduff LP, Bevan HR, Kingsley MI, Owen NJ, Bennett MA, Bunce PJ, Hore AM, Maw JR, Cunningham DJ. Postactivation potentiation in professional rugby players: optimal recovery. J Strength Cond Res, 2007; 21(4): 1134-1138.

Mallander S, Berning J, Pederson S, Adams KJ, DeBeliso M, Stamford B, Maud P. 5RM bench increases medicine ball put distance in trained males and females. Med Sci Sport Exer, 2008; 40(5): S262. 
Markovic G, Dizdar D, Jukic I, Cardinale M. Reliability and factorial validity of squat and countermovement jump tests. J Strength Cond Res, 2004; 18(3): 551-555.

McCurdy K, Langford GA, Cline AL, Doscher M, Hoff R. The reliability of 1-and 3RM tests of unilateral strength in trained and untrained men and women. J Sport Sci Med, 2004; 3(3): 190-196.

Metzger JM, Greaser ML, Moss RL. Variations in cross-bridge attachment rate and tension with phosphorylation of myosin in mammalian skinned skeletal muscle fibers. Implications for twitch potentiation in intact muscle. J Gen Physiol, 1989; 93(5): 855-883.

Moore RL, Stull JT. Myosin light chain phosphorylation in fast and slow skeletal muscles in situ. Am J Physiol-Cell Ph, 1984; 247(5): C462-C471.

NSCA. NSCA Hot Topic: Post-Activation Potentiation (PAP). https://www.nsca.com/Education/Articles/Hot-TopicPost-Activation-Potentiation-\%28PAP\%29/, 2016.

Open Science Collaboration. Estimating the reproducibility of psychological science. Science, 2015; 28: 349(6251): aac4716.

O'Shea P. Quantum Strength Fitness II (Gaining the winning edge). Applied strength training \& conditioning for winning performance, Patrick's Books, Corvallis, OR, USA, 2000.
Rassier DE, MacIntosh BR. Coexistence of potentiation and fatigue in skeletal muscle. Braz J Med Biol Res, 2000; 33: 499-508.

Robbins DW. Postactivation potentiation and its practical applicability: A brief review. J Strength Cond Res, 2005; 19(2): 453-458.

Safrit MJ, Wood TM. Introduction to measurement in physical education and exercise (3rd Ed.) St. Louis, MO: Mosby, 1995.

Smith CE, Lyons B, Hannon JC. A pilot study involving the effect of two different complex training protocols on lower body power. Human Movement, 2014; 15(3): 141-146.

StrengthLevel.Com. Deadlift standards. http://strengthlevel.com/strengthstandards/deadlift $/ \mathrm{kg}$ \#standardsMale. Downloaded 1.17.2016, 2017.

Tagesson SK, Kvist J. Intra-and interrater reliability of the establishment of one repetition maximum on squat and seated knee extension. J Strength Cond Res, 2007; 21(3): 801-807.

Tano G, Bishop A, Berning J, Adams KJ, DeBeliso M. Post activation potentiation in North American high school football players. J Sport Sci, 2016; 4(6): 346-352. 